\title{
Efficacy of Some Ecofriendly Inducers in Controlling Barley Net Blotch El-Nawawy, M. A. ${ }^{1}$; Nabila A. Mosustafa ${ }^{2}$ and Sherin Ph. Ibrahim ${ }^{1}$ \\ 1- Food Sci. Dept., Faculty of Agriculture, Ain Shams Unversity . Egypt . \\ 2- Barley Disease Res. Department , Plant Pathology Reasearch Institute , Agriculture Res Centre, Giza , Egypt.
}

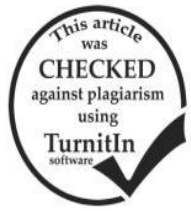

\begin{abstract}
The antifungal activity of resistance inducers ; Mono potassium phosphate $\left(\mathrm{KH}_{2} \mathrm{PO}_{4}\right)$, Chitosan clay Nano composite (ccnc), Humic acid (HA), Sodium meta silicate(Si) Si were tested in controlling Pyrenophoratere anamorph : Drechslerateres the causal organism of net blotch disease of barley . The evaluation was carried out at barley adult plant stages using two rowed hulled Giza 127,Giza 128 and six rowed hulls Giza 129 andGiza 130in greenhouse and open field conditions at two locations ; Itay El-Baroud and Sakha agricultural Research stations in Behaira and KafrElsheik Governorates, Egypt in 2013/ 14 growing season. Under artificial inoculation in greenhouse all concentrations of the four tested inducers were significantly reduced ondisease severity (DS)Chitosan clay Nano composite (ccnc), Mono potassium phosphate $\left(\mathrm{KH}_{2} \mathrm{PO}_{4}\right)$, Humic acid (HA) and Sodium meta silicate(Si)respectively compared with the control. The highest correlation was observed between DS and peroxidase, 1,3-glucanase after 15 days.Under field condition there was similar result of greenhouse. Spraying barley plants of the studied varieties with the tested inducers showed significantly correlation between disease severity and each of thousand kernel weight (TKW), plot weight, total protein , total lipids, total fiber, crude ash and total carbohydrates compared with the control. It is worth mentioning that six rowed hulls barley Giza 129 and Giza 130 were more responsive to the positive effects of inducers compared with two rowed hulled barley Giza 127 andGiza 128 .
\end{abstract}

keywords : barley, Pyrenphorateres, antifungal activity, inducers, net blotch .

\section{INTRODUCTION}

Barley is the fourth most important cereal crop in the world poehlman(1985 ).It has a long history of use as human food and animal feed, of health benefits and for malting and brewing industry in many countries around the world (Malcolmson ,et al., 2005)

There are various forms of barley such as, hulls barley ( a six - rowed ), which is a good alternative to enrich food in developed countries, partriculary due to its significant contents of beneficial substances, such as digestible fiber micronutrients and other components that prevent chronic diseases ( Steffenson ,et al.,1991) .Products with hulls barley, in comparison with products with pure wheat flour, have increased content of beta - glucans , i-e by more than the fold . hulled barley ( two - rowed) is used mostly for malt production , Noworolnik , ( 2004) studied various forms of barley and indicated a definitely higher content of protein and a lower content of ash and crude fiber in hulles forms than in the hulled ones

Net Blotch ( BNB) caused by pyrenphora teres anamorph : Drechslerateres, is one of the major disease of barley in Egypt and most of the cereal growing regions of the world .Under warm and humid conditions expression of barley net blotch ( BNB) Tekauz, (2003) disease symptoms can increase rapidly, causing substantial grain yield loss $27 \%$ on an average and up to $34 \%$ when it is sever (Yitbarek and Wudneh ,1985).Typical yield losses due to net blotch nearing $100 \%$ in some highly susceptible barley cultivars. The disease is wide- spread in Northern Egypt, common in Middle Egypt while not detected in Assiout(Upper Egypt). The disease was also found to be of widespread along the northern coast. (Faten ElNashar,1983)Disease resistance has been a major strategy in controlling net blotch of barley . Several control methods against P.teres had been recommended, such as crop rotation, the application of fungicides and the use of resistant cultivars. The use of genetic resistance is the favoured for controlling this disease ; however ; it is complicated by the existence of several pathotypes of the pathogen (Boungab , et al.,2012). An alternative control approach against net blotch would be based on economically and technically feasible and environmentally safe strategy . Many investigations have been made to understand the physiological and biochemical basis of induced systemic resistance (ISR). Much of this knowledge is due to the identification of a number of chemical and biological elicitors, some of them are commercially available for use in conventional Agriculture ( Gary and Robert , 2004). Several natural and synthetic chemical agents have been described as activators of defense - related processes when applied to plants . Some of these activators may have potential application in Agriculture ( Yamaguchi, 1998 ) .

Monopotassium phosphate is the formulation with the lowest salt index and thus the foliar fertilizer of choice for many crops. It is an excellent and fast source of Pand $\mathrm{K}$ when applied as a foliar fertilizer . Additionally, it controls powdery mildew in fruit trees and grapevines ( Reuveni and Reuveni, 2001 ).

Application of humic acids ( HA) has several benefits and agriculturists all over the world are accepting humic acids as an integral part of their fertilizer program . Humic acid is one of major component of humus .Humates are natural organic substances, high in humic acid and containing most of known trace minerals necessary to the development of plant life. Studies of the positive effect of humic substance on plant growth have demonstrated the importance of optimum mineral supply independent of nutrition .(Yildirim , 2007).

Chitin and chitosan arenaturally - occurring compound that have potential in agriculture with regard to controlling plant diseases. These molecules were shown to display toxicity and inhibit fungal growth and development. Fragments from chitin and chitosan are known to have eliciting activities leading to a variety of defense responses in host plants in response to microbial infections, including the accumulation of phytoalexins, pathogen- related (PR) proteins and proteinase inhibitors, lignin synthesis and callose formation 
.Based on these and other proprieties that help strengthen host plant defenses, interest has been growing in using them in agricultural system to reduce the negative impact of diseases on yield and quality of crops . (El Hadrami et al., 2010).Si can activate the expression of defense related genes and mayplay important role in the transduction of plant stress signal such as salicylic acid ,Jasmonic acid and ethylene (Kunzheng et al., 2009).

The objectives of this study were to evaluated the response of some two - rowed ( hulled barley ) and six rowed ( hulls barley ) genotypes to infection caused by pyrenphora teres, and to estimate the improvement in of yield component and chemical composition when some safe resistance inducers were used for controlling barley net Blotch diseases .

\section{MATERIALS AND METHODS}

\section{1) Plant materials}

The grains of Egyptian Barley ( HordiumVulgare) cultivars ( Giza 127 andGiza 128 ) as two rowed hulled, (Giza 129 and Giza 130 ) as six rowed hullesswere obtained from Barley Research Section ,field cropInstitute, Agriculture Research Center (ARC) Giza , Egypt

Table (1) shows the pedigree of barley cultivars under investigation

\begin{tabular}{llc}
\hline No. & Cultivars & Pedigree \\
\hline 1 & Giza 127 & "w 12291'/' Bags'// Harmal- 02 and 'W 12291'/4/' 11012-2'/'70 -22425'/3/'Apm'/'IB65'//'A16' \\
2 & Giza 128 & "w 12291'/' Bags'// Harmal- 02 and 'W 12291'/4/' 11012-2'/'70 -22425'/3/'Apm'/'IB65'//'A16' \\
3 & Giza 129 & 'DeirAlla 106/Cell//As46/Aths*2', \\
4 & Giza 130 & 'Comp. cross 229//Bco Mr/DZ02391/3/DeirAlla 106", \\
\hline
\end{tabular}

2) source of four chemical inducers:

Mono potassium phosphate $\left(\mathrm{KH}_{2} \mathrm{PO}_{4}\right)(50 \& 100$ $\mu \mathrm{g} / \mathrm{ml})$, Chitosan clay nano composite (ccnc) (15 \&30 $\mathrm{mg} / \mathrm{ml})$, Humic acid $(1.5 \& 2.5 \mathrm{~m} / \mathrm{l})$, and Sodium meta silicate $(\mathrm{Si})(1 \& 1.5 \mathrm{~g} / \mathrm{l})$, were tested to evaluate their capabilities to induce resistance against net blotch (BNB) caused by pyrenphora teres anamorph : Drechslera teres.

Mono potassium phosphate $\left(\mathrm{KH}_{2} \mathrm{PO}_{4}\right)$, Chitosan clay Nano composite and Humicacid,were obtained from central Laboratory of Organic Agriculture , Agriculture Res. Center ,Giza , Egypt

Sodium meta silicate ( $\mathrm{Si}$ ) was obtained from Sigma Aldrich Chemical Co. ( St. Louis, MO, USA) .

The following experiments were conducted under greenhouse condition (artificial infection) and field conditions (natural infection )

3- Greenhouse experiments :

a-Isolation of the pathogen:

Samples of naturally infected barley leaves showing net blotch symptoms with necrotic lesions were collected from different locations. Small pieces ( $5 \mathrm{~mm})$ were cut from each sample and sterilized with sodium hypochlorite $1 \%$ for $1 \mathrm{~min}$ and dried between folds of sterilized filter papers and placed on potato dextrose ager plats ( PDA) supplemented with streptomycin sulphate $(100 \mathrm{mg} / \mathrm{ml})$. Petri dishes were incubated at $24^{\circ} \mathrm{C}$ for $48-72$ hours. After 4 days incubation they were kept at $4{ }^{\circ} \mathrm{C}$ until use in further experiments.

Barley plants were grown in the clay pots $(30 \mathrm{~cm}$ diameter ) in a greenhouse at $20 \pm 2^{\circ} \mathrm{C}$ all the cultural practices applied. Adult barley plants( 70 days old ) with three replicates were assigned for each treatment were sprayed with one of the following chemical compounds : Mono potassium phosphate $\left(\mathrm{KH}_{2} \mathrm{PO}_{4}\right)(50 \& 100 \mu \mathrm{g} / \mathrm{ml})$, Chitosan clay Nano composite (ccnc)( $15 \& 30 \mathrm{mg} / \mathrm{ml})$, Humic $\operatorname{acid}(1.5 \& 2.5 \mathrm{~m} / \mathrm{l})$, and Sodium meta silicate(Si) ( $1 \& 1.5 \mathrm{~g} / \mathrm{l})$,control plants were only sprayed with distilled water. five days after treatment, plants were inoculated with Drechslera teres.by spraying a suspension of the fungus adjusted to a concentration of $2 \times 10^{4}$ conidia / ml (Gupta et al., 2003) .

The inoculated plants were incubated in the dark for $48 \mathrm{~h}$ at $20 \pm 2 \mathrm{C}^{\circ}$ and $100 \%$ relative humidity ; they were then transferred to a growth chamber maintained at $20 \pm 2 \mathrm{C}^{\circ}$ and $70-80 \% \mathrm{RH}$ with $12 \mathrm{hr}$ daylight per day . Disease severity was determined as the percentage of infected leaf area 9 days after inoculation.

\section{b-Disease assessments}

Net blotch were carried out 12-14 d after inoculation (DAI). The net-like necrosis was assessed visually using the rating scale of Tekauz (1978) as illustrated in Figure

Inoculated barley plants were estimated and calculated using formula suggested by Towsend and Heuberger, (1943) as flows :

$$
\text { Disease severity }(\%)=\begin{aligned}
& \sum(\text { NPC X CR }) \\
& \hdashline-N^{-}
\end{aligned} \quad \times 100
$$

Where : NPC $=$ no. of plants in each class rate $\mathrm{CR}=$ Class Rate NIP $=$ No. of tested plants. MSC = Maximum severity class rate .

\section{Determination of enzyme activities : \\ 1. Peroxidase activity :}

Leaf samples of two rowed and six rowed barley cultivars were used to determine the activity of peroxidise (PO).the extraction procedure was essentially based on the methods described by Biles and Martyn, (1993).Peroxidase activity was expressed as changes in absorbance $\min ^{-1} \mathrm{~g}^{1}$ fresh weight at $425 \mathrm{~nm}$ by using spectrophotometer ( Unico - 2100).

\section{2- Extraction and assay of $\beta-1,3$ glucanase :}

The method of Pan et al.( 1991 ) was used to determine $\beta-1,3$ glucanase activity . Laminarine was used as substrate and dinitro salicylic acid as reagent to measure reducing sugars .the method was carried out as $0.5 \mathrm{ml}$ of enzyme extract was added to $0.5 \mathrm{ml}$ of $0.05 \mathrm{M}$ of potassium acetate buffer ( $\mathrm{pH} 5$ ) containing $2 \%$ laminarin. The mixture was incubated at $50^{\circ} \mathrm{C}$ for 60 minutes. The reaction was stopped by adding $1 \mathrm{ml}$ of dinitrosalicylic acid reagent and heating the tubes for 5 
minutes at $100^{\circ} \mathrm{C}$. The tubes were cooled and $3 \mathrm{ml}$ of distilled water were added before assay. The optical density was red at $500 \mathrm{~nm} . \beta-1,3$ glucanase activity was expressed as $\mathrm{mM}$ glucose equivalent released /gram fresh weight tissues /60 minutes.

\section{Field experiments}

Field experiments were carried out under natural infection at experimental farms of Itay EL-Baroud (Behaira Governorate) and Sakha (KafrElsheik Governorate), in 2013/14 growing season. The experiment was conducted in a randomized complete block design (RCBD), with three replicates to each treatment .The plot size was $3 \times 3.5 \mathrm{~m} 2$ (1/400 Fadden) and each plot included 6 rows with $20 \mathrm{~cm}$ between rows. Each row was sown by $5 \mathrm{~g}$ barley grains .The tested elicitors were prepared by mixing with sterile distilled water containing $0.01 \%$ Tween 20 to obtain the desired final concentration .In control treatments, sterilized distilled water and Tween 20 were used instead of the inducers. The different treatments with four inducers were sprayed after 70 days from planting.to determine the effect of different concentrations of four inducers while the control plots were sprayd with distilled water .

Yield components and chemical analyzed:

At maturity plants were harvested and Grain yield / plot $(\mathrm{kg})$ and one thousand - kernel weight (TKW) (g) were determined. samples from different treatments to all varieties were chemically analyzed for protein, lipids, fiber, ash and carbohydrates according to the methods described in the A.O.A.C ( 2005 ).

Disease assessment of field :

The reaction of barley plants to net blotch was recorded at growth stage ; 10.5(Large, 1954)according to double - digit scale 00-99. The first digit gives the relative height of the disease, while the second digit shows the disease severity as a percentage of leaf area affected in terms of $0-9$, where $0=0 \%, 2=20 \%$, $30=3 \%$, ( Eyal et al., 1987 ) .

Table (2) The observation and response of barley plants to net blotch infection at adultplant stage :

\begin{tabular}{llcc}
\hline No. & Reaction & Observation & Response value \\
\hline 1 & No disease & HR & 00 \\
2 & Resistant & R & 0.1 \\
3 & Resistant - Moderately resistant & R- MR & 0.2 \\
4 & Moderately resistant & MR & 0.3 \\
5 & Mod. Res. - Mod.Sucep. & MR - MS & 0.4 \\
6 & Moderately susceptible & MS & 0.6 \\
7 & Mod.Susc - Suscep & MS - S & 0.8 \\
8 & Susceptible & S & 0.9 \\
9 & Highly susceptible & HS & 1.0 \\
\hline
\end{tabular}

\section{Statistical analysis}

All experiments were carried out as a randomized complete block design with three replicates.Data were subjected to analysis of variance (ANOVA) by using MSTAT-C statistical package and the least significant differences (LSD was used to compare means .Correlation analysis was performed with SPSS 6.0 statistical package.

\section{RESULTS}

\section{Green house experiments}

Anova of Table (3) showed highly significant effects for inducers, concentrations and varieties while all the interactions between concentration, variety and inducers were non Significant. Data in Table 4 show that the effect of spraying barley varieties with different inducers on net blotch disease severity . A significant reduction of the disease was recorded to all inducers and at higher concentrations .chitosan clay was the best treatment in reducing net blotch severity followed by $\mathrm{KH}_{2} \mathrm{PO}_{4}$, Humic acid and $\mathrm{Si}$ respectively . Moreover, reduction of net blotch severity was higher on cultivars Giza 130 followed by Giza 129 ( hulls barley ) than varietiesGiza 128 followed by Giza 127 and control .

Table (3) Analysis of variance of disease severity (DS) of net blotch disease when mono potassium phosphate $\left(\mathrm{KH}_{2} \mathrm{PO}_{4}\right)$, Chitosan nanoparticles , Humic acid and Silicon were applied at three concentrations on four barley cultivars

\begin{tabular}{lcccc}
\hline Source of variation & D. F. & M.S. & F.VALU & P > F \\
\hline Concentration ( c ) & 2 & 4.709 & 20.0345 & 0.0000 \\
Inducer ( I ) & 3 & 1.480 & 6.2943 & 0.0006 \\
C X I & 6 & 0.488 & 2.0745 & 0.0632 \\
Variety ( v ) & 3 & 1.335 & 5.6784 & 0.0013 \\
C X V & 6 & 0.210 & 0.8933 & \\
I X V & 9 & 0.147 & 0.7408 & \\
C X V X I & 18 & 0.069 & 0.2919 & \\
Error & 96 & 0.235 & & \\
\hline
\end{tabular}

Table (4) Effect of spraying barley plants on severity of net blotch disease severity under greenhouse condition .

\begin{tabular}{lcccccc}
\hline \multirow{2}{*}{ substance } & \multicolumn{6}{c}{ Disease severity } \\
& Conc. & Giza & Giza & Giza & Giza & General \\
& & $\mathbf{1 2 7}$ & $\mathbf{1 2 8}$ & $\mathbf{1 2 9}$ & $\mathbf{1 3 0}$ & mean \\
\hline \multirow{3}{*}{$\mathrm{KH}_{2} \mathrm{PO}_{4}$} & $50 \mu \mathrm{g} / \mathrm{ml}$ & 43.3 & 36.6 & 20.0 & 13.3 & 29.98 \\
& $100 \mu \mathrm{g} / \mathrm{ml}$ & 30.0 & 16.6 & 13.3 & 10.0 & 17.48 \\
& $\mathrm{Mean}$ & 36.65 & 26.6 & 16.65 & 11.65 & 23.73 \\
$\mathrm{CCNC}$ & $15 \mathrm{mg} / \mathrm{ml}$ & 36.6 & 33.3 & 23.3 & 10.0 & 25.8 \\
& $30 \mathrm{mg} / \mathrm{ml}$ & 20.0 & 13.3 & 10.0 & 0.0 & 10.83 \\
& Mean & 28.3 & 23.3 & 16.65 & 5.0 & 18.32 \\
Humic acid & $1.5 \mathrm{~m} / 1$ & 50.0 & 40.0 & 26.6 & 16.6 & 33.3 \\
& $2.5 \mathrm{~m} / 1$ & 30.0 & 26.6 & 16.6 & 13.3 & 21.63 \\
& Mean & 40.0 & 33.3 & 21.6 & 14.95 & 27.47 \\
Si & $1.0 \mathrm{~g} / 1$ & 53.3 & 43.3 & 30.0 & 23.3 & 37.48 \\
& $1.5 \mathrm{~g} / 1$ & 56.6 & 30.0 & 23.3 & 16.6 & 31.63 \\
& Mean & 54.95 & 36.65 & 26.65 & 19.95 & 34.56 \\
control & & 85.0 & 80.0 & 60.0 & 50.0 & 68.75 \\
& Genral & 40.81 & 29.96 & 17.48 & 12.89 & 29.30 \\
\hline
\end{tabular}

Activity of peroxidase and $\beta 1-3$ gluconseenzymes :

Four treatments were used to study their effect on peroxidase and $\beta 1-3$ gluconse activities which play a main role as plant defence mechanisms. Results in table(5 and 6) indicate that all treatment stimulated enzymes activity at second concentration after 15 day .

The highest activity was obtained with chitosan followed by $\mathrm{KH}_{2} \mathrm{PO}_{4}$ compared to other treatment. Meanwhile, in $\beta 1-3$ gluconse the highest activity was obtained with $\mathrm{KH}_{2} \mathrm{PO}_{4}$ followed by chitosan , Humic acid and silcon as compared with control .

VarietiesGiza 130 and Giza 129 (hulls barley) were highly affected with enzymes activities than Giza 128 and Giza 127 ( hulled barley ) . 
Table ( 5 ) Effect of spraying different inducers on peroxidase activity of barley varieties ( Ciza 127 ,Ciza 128 , Ciza 129 andCiza 130 ) under greenhouse conditions (artificial infection)

\begin{tabular}{|c|c|c|c|c|c|c|c|c|c|c|}
\hline \multirow[b]{2}{*}{ substance } & \multirow[b]{2}{*}{ Con. } & \multicolumn{6}{|c|}{ *Peroxidase Activity \% } & \multicolumn{2}{|c|}{ Ciza 130} & \multirow{2}{*}{ Mean } \\
\hline & & 3day & 15 day & 3day & 15 day & 3day & 15 day & 3day & 15 day & \\
\hline & $50 \mu \mathrm{g} / \mathrm{ml}$ & 0.65 & 1.160 & 1.28 & 2.45 & 1.79 & 2.020 & 1.85 & 1.99 & 1.64 \\
\hline $\mathrm{KH}_{2} \mathrm{PO}_{4}$ & $100 \mu \mathrm{g} / \mathrm{ml}$ & 0.72 & 1.22 & 1.92 & 3.68 & 1.99 & 2.34 & 2.42 & 3.83 & 2.27 \\
\hline Mean & & 0.69 & 1.19 & 1.6 & 3.06 & 1.89 & 2.18 & 2.14 & 2.91 & 1.96 \\
\hline cons & $15 \mathrm{mg} / \mathrm{ml}$ & 0.77 & 1.43 & 1.20 & 2.21 & 1.43 & 2.68 & 1.22 & 2.40 & 1.66 \\
\hline $\operatorname{ccnc}$ & $30 \mathrm{mg} / \mathrm{ml}$ & 0.96 & 1.93 & 1.90 & 3.18 & 1.95 & 3.30 & 2.40 & 3.60 & 2.40 \\
\hline Mean & & 0.87 & 1.68 & 1.55 & 2.69 & 1.69 & 2.99 & 1.81 & 3.0 & 2.03 \\
\hline Humic acid & $1.5 \mathrm{~m} / 1$ & 0.55 & 0.97 & 0.69 & 1.09 & 0.98 & 1.05 & 0.52 & 1.80 & 0.95 \\
\hline Humic acid & $2.5 \mathrm{~m} / 1$ & 0.81 & 1.57 & 0.89 & 1.44 & 1.12 & 2.04 & 0.98 & 2.02 & 1.35 \\
\hline Mean & & 0.68 & 1.27 & 0.79 & 1.26 & 1.05 & 1.55 & 0.75 & 1.91 & 1.15 \\
\hline $\mathrm{Si}$ & $1.0 \mathrm{~g} / 1$ & 0.62 & 0.92 & 0.72 & 1.07 & 0.97 & 1.03 & 1.10 & 1.72 & 1.01 \\
\hline S1 & $1.5 \mathrm{~g} / 1$ & 0.89 & 1.24 & 0.85 & 1.40 & 1.12 & 2.02 & 1.90 & 1.40 & 1.35 \\
\hline Mean & & 0.76 & 1.08 & 0.79 & 1.24 & 1.05 & 1.53 & 1.5 & 1.56 & 1.18 \\
\hline control & & 0.45 & 0.80 & 0.58 & 0.9 & 0.65 & 0.81 & 0.72 & 0.98 & 0.73 \\
\hline LSD $p \leq 0.05$ & & 0.18 & 0.71 & 0.78 & 0.92 & 0.79 & 0.89 & 0.78 & 1.2 & \\
\hline
\end{tabular}

*peroxidase activity expressed as absorbance/3.0 min $1.0 \mathrm{~g}$ fresh weight .

Table (6) Effect of spraying different inducers on $\beta 1-3$ gluconse activity of barley varieties (Ciza 127 ,Ciza 128 , Ciza 129,Ciza 130 ) under greenhouse conditions (artificial infection)

\begin{tabular}{|c|c|c|c|c|c|c|c|c|c|c|}
\hline \multirow[b]{2}{*}{ substance } & \multirow[b]{2}{*}{ Con. } & \multicolumn{6}{|c|}{$* \beta 1-3$ gluconseActivity $\%$} & \multicolumn{2}{|c|}{ Ciza 130} & \multirow[b]{2}{*}{ Mean } \\
\hline & & 3day & 15 day & 3day & 15 day & 3day & 15 day & 3day & 15 day & \\
\hline & $50 \mu \mathrm{g} / \mathrm{ml}$ & 0.51 & 0.69 & 0.29 & 0.47 & 0.35 & 0.51 & 0.42 & 0.55 & 0.47 \\
\hline $\mathrm{KH}_{2} \mathrm{PU}_{4}$ & $100 \mu \mathrm{g} / \mathrm{ml}$ & 0.713 & 0.93 & 0.55 & 0.65 & 0.48 & 0.68 & 0.52 & 0.79 & 0.66 \\
\hline Mean & & 0.61 & 0.81 & 0.42 & 0.56 & 0.41 & 0.59 & 0.47 & 0.67 & 0.56 \\
\hline & $15 \mathrm{mg} / \mathrm{ml}$ & 0.20 & 0.41 & 0.18 & 0.43 & 0.22 & 0.44 & 0.33 & 0.49 & 0.33 \\
\hline cenc & $30 \mathrm{mg} / \mathrm{ml}$ & 0.32 & 0.55 & 0.45 & 0.60 & 0.35 & 0.66 & 0.45 & 0.68 & 0.50 \\
\hline Mean & & 0.26 & 0.48 & 0.31 & 0.51 & 0.28 & 0.55 & 0.39 & 0.58 & 0.42 \\
\hline Humic acid & $1.5 \mathrm{~m} / 1$ & 0.197 & 0.57 & 0.12 & 0.26 & 0.14 & 0.41 & 0.29 & 0.45 & 0.30 \\
\hline Humic acid & $2.5 \mathrm{~m} / 1$ & 0.28 & 0.71 & 0.34 & 0.48 & 0.46 & 0.64 & 0.39 & 0.60 & 0.48 \\
\hline Mean & & 0.23 & 0.64 & 0.23 & 0.37 & 0.30 & 0.52 & 0.34 & 0.52 & 0.39 \\
\hline $\mathrm{Si}$ & $1.0 \mathrm{~g} / 1$ & 0.12 & 0.32 & 0.08 & 0.25 & 0.22 & 0.34 & 0.25 & 0.35 & 0.18 \\
\hline S1 & $1.5 \mathrm{~g} / 1$ & 0.18 & 0.41 & 0.32 & 0.45 & 0.35 & 0.49 & 0.36 & 0.59 & 0.39 \\
\hline Mean & & 0.15 & 0.36 & 0.20 & 0.35 & 0.28 & 0.83 & 0.30 & 0.47 & 0.36 \\
\hline contro & & 0.10 & 0.09 & 0.21 & 0.18 & 0.18 & 0.23 & 0.11 & 0.14 & 0.15 \\
\hline LSD $p \leq 0.05$ & & 0.15 & 0.17 & 0.15 & 0.17 & 0.16 & 0.17 & 0.15 & 0.17 & \\
\hline
\end{tabular}

* $\beta$ - 1,3gluconase activity expressed as $\mathbf{m M}$ glucose equivalent released / gram fresh weight/60 min .

Data in Table 7 show that the disease severity

(DS) was significantly and negatively correlated with each of the biochemical traits . the highest correlation was observed between DS and $\beta 1,3$-glucanas,peroxidase ,after 15 day rating -0.531 and- 0.351 respectively.

Table (7) Correlation between disease severity ( DS) of net blotch disease and activities of peroxidase andß1,3-glucanas under greenhouse condition (artificial infection)

\begin{tabular}{|c|c|c|c|c|}
\hline \multicolumn{5}{|c|}{ Variable } \\
\hline Peroxidase $3 \mathrm{~d}$. & & & & \\
\hline Peroxidase $15 d$ & $0.827 * *^{\mathrm{a}}$ & & & \\
\hline$\beta 1,3$-glucanas3d & $0.509 * *$ & $0.595 * *$ & & \\
\hline$\beta 1,3$-glucanas 15 & $0.509 * *$ & $0.628 * *$ & $0.841 * *$ & \\
\hline D.S & $-0.321 * *$ & $-0.351 * *$ & $-0.343 * *$ & $-0.531 * *$ \\
\hline
\end{tabular}

Field experiments :

Data in Table 8 showed highly significant correlation between disease severity and each of 1000 kernel weight, plot weight, total protein , total lipids, total fiber, crude ash and total carbohydrates. The highest negative correlation between disease severity and 1000 kernel weight, plot weight and total fiber which rating $-0.648,-0.493$ and -0.362 respectively implies that the substance, which significantly decreased disease severity will induce significant increase in these trails .
Data Table 9 showed highly significant correlation between disease severity and each of 1000 kernel weight , plot weight, total protein, total lipids , total fiber, crude ash and total carbohydrates. The highest negative correlation between disease severity and 1000 kernel weight plot weight and total fiber which rating $-0.716,-0.688$ and -0.501 respectively implies that the substance, which significantly decreased disease severity will induce significant increase in 1000 kernel weight, plot weight and total fiber . 
Table (8 ) Correlation between disease severity of net blotch, agronomic traits and technological traits under field conditions in Etay -el baroud location .

\begin{tabular}{|c|c|c|c|c|c|c|c|c|}
\hline No. & \multicolumn{8}{|c|}{ Variable } \\
\hline 1 & 1000 kernel weight & & & & & & & \\
\hline 2 & plot weight & $0.539^{* * a}$ & & & & & & \\
\hline 3 & total protein & -0.067 & $-0.435^{* *}$ & & & & & \\
\hline 4 & total lipids & 0.058 & -0.011 & $0.558^{* *}$ & & & & \\
\hline 5 & total fiber & $0.256^{* *}$ & -0.039 & $0.797^{* *}$ & $0.836^{* * *}$ & & & \\
\hline 6 & crude ash & -0.012 & $-0.253^{* *}$ & $0.790^{* *}$ & $0.721^{\text {*** }}$ & $0.844^{* *}$ & & \\
\hline 7 & total carbohydrates & -0.138 & -0.093 & $0.221^{* * *}$ & $0.819^{* *}$ & $0.475^{* * *}$ & $0.498^{* *}$ & \\
\hline 8 & disease severity & $-0.648^{* *}$ & $-0.493^{* *}$ & -0.061 & -0.127 & $-0.362^{* *}$ & -0.035 & 0.112 \\
\hline
\end{tabular}

${ }^{\text {a) }}$ linear correlation coefficient $(r)$ is significant at $p \leq 0.01(* *)$ or $p \leq 0.05(*)$ and $n=48$

The obtained results revealed that all the tested treatments reduced significantly disease severity compared to the control .( table 10 and 11) The second concentration showed the best effected against the disease . In this respect $\mathrm{KH}_{2} \mathrm{PO}_{4}$ was the most effective treatment, where it recorded the lowest degree of disease severity followed by chitosan, humic acid and $\mathrm{Si}$.All tested genotypes showed also the highest degree of disease reduction over the highest degree of disease reduction over the control , 64.2-81.5\% in Giza 127, 51.1 - $74.2 \%$ Giza 128 45.0-86.0\% in Giza 129 and $62.8-81.1 \%$ in Giza 130 at Etay El boarod location . Also , at Sakha, net blotch control over non- inducers applied treatment ranged from $63.6-85.4 \%$ in Giza $127,54.4-81.1 \%$ in Giza $128,37.5-85.0 \%$ in Giza 129 and $53.3-80.0 \%$ in Giza 130.

Table (9 ) correlation between disease severity of net blotch, agronomic traits technological traits under field condition in Sakha location .

\begin{tabular}{|c|c|c|c|c|c|c|c|c|}
\hline No. & variable & 1000 Kernel weight & plot weight & $\begin{array}{l}\text { Variable } \\
\text { total protein }\end{array}$ & total lipids & total fiber & crude ash & totalcarbo. \\
\hline 1 & 1000 kernel weight & & & & & & & \\
\hline 2 & plot weight & $0.739^{* * a}$ & & & & & & \\
\hline 3 & total protein & 0.089 & -0.005 & & & & & \\
\hline 4 & total lipids & $0.183^{*}$ & 0.144 & $0.580^{* *}$ & & & & \\
\hline 5 & total fiber & $0.355^{* *}$ & $0.282^{* * *}$ & $0.789^{* *}$ & $0.862^{* *}$ & & & \\
\hline 6 & crude ash & 0.053 & 0.004 & $0.784^{* * *}$ & $0.724^{* * *}$ & $0.835^{* *}$ & & \\
\hline 7 & total carbohydrates & -0.106 & $-0.171^{*}$ & $0.224^{* * *}$ & $0.807^{* *}$ & $0.508^{* *}$ & $0.502^{* *}$ & \\
\hline 8 & disease severity & $-0.716^{* *}$ & $-0.688^{* *}$ & $-0.400^{* *}$ & $-0.263^{* *}$ & $-0.501^{* *}$ & $-0.243^{* *}$ & 0.124 \\
\hline
\end{tabular}

Table (10) Effect of spraying barley genotype with some inducers on disease severity plants cvs. (Giza 127, Giza 128) as two rowed hulled and (Giza 129, Giza130 ) as six rowed Hullesunder field conditions during 2013/2014 growing season in Etay El boarod location

\begin{tabular}{|c|c|c|c|c|c|c|c|c|c|}
\hline \multirow{2}{*}{ Sub. } & \multirow{2}{*}{ Con. } & \multicolumn{2}{|c|}{ Giza 127} & \multicolumn{2}{|c|}{ Giza 128} & \multicolumn{2}{|c|}{ Giza 129} & \multicolumn{2}{|c|}{ Giza 130} \\
\hline & & DS* & Decrease \% & DS & Decrease \% & DS & Decrease \% & DS & Decrease \% \\
\hline \multirow{2}{*}{$\mathrm{KH}_{2} \mathrm{PO}_{4}$} & $50 \mu \mathrm{g} / \mathrm{ml}$ & 15.6 & 76.0 & 13.0 & 71.0 & 12.7 & 74.6 & 10.0 & 71.4 \\
\hline & $100 \mu \mathrm{g} / \mathrm{ml}$ & 12.0 & 81.5 & 11.6 & 74.2 & 7.0 & 86.0 & 6.6 & 81.1 \\
\hline \multirow{2}{*}{$\mathrm{CCNC}$} & $15 \mathrm{mg} / \mathrm{ml}$ & 16.6 & 74.5 & 15.0 & 66.6 & 13.3 & 73.4 & 11.0 & 68.5 \\
\hline & $30 \mathrm{mg} / \mathrm{ml}$ & 13.3 & 79.5 & 13.0 & 71.0 & 7.8 & 84.4 & 7.2 & 79.4 \\
\hline \multirow{2}{*}{ humic acid } & $1.5 \mathrm{~m} / 1$ & 20.0 & 69.0 & 17.2 & 61.7 & 27.0 & 45.0 & 12.0 & 65.7 \\
\hline & $2.5 \mathrm{~m} / 1$ & 15.0 & 76.9 & 15.6 & 65.3 & 16.7 & 66.6 & 8.4 & 76.0 \\
\hline \multirow{2}{*}{$\mathrm{Si}$} & $1.0 \mathrm{~g} / 1$ & 23.3 & 64.2 & 22.0 & 51.1 & 23.3 & 53.4 & 13.0 & 62.8 \\
\hline & $1.5 \mathrm{~g} / 1$ & 17.6 & 72.9 & 20.0 & 55.5 & 17.0 & 66.0 & 9.3 & 73.4 \\
\hline control & & 65.0 & ------ & 45.0 & ------ & 50.0 & ----- & 35.0 & -------- \\
\hline LSD $\quad \mathrm{p} \leq 0.05$ & & 2.3 & & 2.3 & & 2.3 & & 2.3 & \\
\hline
\end{tabular}

*DS : disease severity

Table (11 ) : Effect of spraying barley varieties with some inducers on disease severity plants cvs. under field conditions during 2013/2014 growing season in Sakha location

\begin{tabular}{|c|c|c|c|c|c|c|c|c|c|}
\hline \multirow{2}{*}{ substance } & \multirow{2}{*}{ Con. } & \multicolumn{2}{|c|}{ Giza 127} & \multicolumn{2}{|c|}{ Giza 128} & \multicolumn{2}{|c|}{ Giza 129} & \multicolumn{2}{|c|}{ Giza 130} \\
\hline & & *DS & Decrease \% & DS & Decrease \% & DS & Decrease \% & DS & Decrease \% \\
\hline \multirow{2}{*}{$\mathrm{KH}_{2} \mathrm{PO}_{4}$} & $50 \mu \mathrm{g} / \mathrm{ml}$ & 13.0 & 76.4 & 10.2 & 77.3 & 11.0 & 72.5 & 10 & 66.6 \\
\hline & $100 \mu \mathrm{g} / \mathrm{ml}$ & 8.0 & 85.4 & 8.5 & 81.1 & 6.0 & 85.0 & 6.0 & 80.0 \\
\hline \multirow{2}{*}{$\mathrm{CCNC}$} & $15 \mathrm{mg} / \mathrm{ml}$ & 15.0 & 72.7 & 13.5 & 70.0 & 13.5 & 58.8 & 11 & 63.3 \\
\hline & $30 \mathrm{mg} / \mathrm{ml}$ & 9.0 & 83.6 & 9.5 & 78.8 & 8.0 & 80.0 & 7.9 & 73.6 \\
\hline \multirow{2}{*}{ humic acid } & $1.5 \mathrm{~m} / 1$ & 20.0 & 63.6 & 19.4 & 56.8 & 23.0 & 42.5 & 13 & 56.6 \\
\hline & $2.5 \mathrm{~m} / 1$ & 12.0 & 78.2 & 11.2 & 75.1 & 16.0 & 60.0 & 9.9 & 67.0 \\
\hline \multirow{2}{*}{$\mathrm{Si}$} & $1.0 \mathrm{~g} / 1$ & 18.0 & 67.2 & 20.5 & 54.4 & 25.0 & 37.5 & 14 & 53.3 \\
\hline & $1.5 \mathrm{~g} / 1$ & 14.0 & 74.5 & 13.5 & 70.0 & 18.0 & 55.0 & 11 & 63.3 \\
\hline control & & 55.0 & ------ & 45.0 & ------ & 40.0 & ----- & 30 & ------ \\
\hline LSD $p \leq 0.05$ & & 1.8 & & 1.8 & & 1.8 & & 1.8 & \\
\hline
\end{tabular}

*DS : disease severity 
2- yield component :

Data in Table 12 at Etay El boarod location show that all tested treatments significantly improved barley 1000 grain weight and plot weight over the control. $\mathrm{KH}_{2} \mathrm{PO}_{4}$ treatment was more effective in increasing crop parameters followed by chitosan ,humic acid and $\mathrm{Si}$, respectively. The second concentration showed the best effected on the crop parameters compared to the control. On the other hand genotypes Giza 129 and Giza 130 (Hull- less ) barley were the most improving in 1000 grain weight and plot weight than genotypes Giza 128and Giza 127 ( Hulled barley ).

Table (12 ) : Effect of inducers on yield components on Giza 127and Giza 128 , Giza 129 and Giza 130 under field conditions during 2013/2014 growing season in Etay El boarod location .

\begin{tabular}{|c|c|c|c|c|c|c|c|c|c|}
\hline \multirow[b]{2}{*}{ substance } & \multirow[b]{2}{*}{ Con. } & \multicolumn{4}{|c|}{ Giza 127} & \multicolumn{4}{|c|}{ Giza 128} \\
\hline & & $\begin{array}{l}1000 \text { Grain } \\
\text { Weight (g) }\end{array}$ & $\begin{array}{c}\text { Increase } \\
\%\end{array}$ & $\begin{array}{c}\text { Plot } \\
\text { Weight (kg) }\end{array}$ & $\begin{array}{c}\text { Increase } \\
\%\end{array}$ & $\begin{array}{r}1000 \text { Grain } \\
\text { Weight (g) }\end{array}$ & $\begin{array}{c}\text { Increase } \\
\%\end{array}$ & $\begin{array}{c}\text { Plot } \\
\text { Weight (kg) }\end{array}$ & $\begin{array}{c}\text { Increase } \\
\%\end{array}$ \\
\hline \multirow{2}{*}{$\mathrm{KH}_{2} \mathrm{PO}_{4}$} & $50 \mu \mathrm{g} / \mathrm{ml}$ & 51.9 & 21.1 & 4.5 & 45.2 & 52.8 & 20.5 & 6.8 & 78.9 \\
\hline & $100 \mu \mathrm{g} / \mathrm{ml}$ & 57.3 & 34.5 & 4.9 & 58.1 & 58.3 & 33 & 7.5 & 97.4 \\
\hline \multirow{2}{*}{$\mathrm{CCNC}$} & $15 \mathrm{mg} / \mathrm{ml}$ & 51.3 & 20.4 & 4.3 & 38.7 & 51.6 & 17.8 & 5.9 & 55.3 \\
\hline & $30 \mathrm{mg} / \mathrm{ml}$ & 56.3 & 32.2 & 4.8 & 32.3 & 57 & 30.1 & 6.3 & 65.7 \\
\hline \multirow{2}{*}{ humic acid } & $1.5 \mathrm{~m} / 1$ & 50.7 & 19 & 4.1 & 32.3 & 50.8 & 15.9 & 5.8 & 52.6 \\
\hline & $2.5 \mathrm{~m} / 1$ & 55.9 & 31.2 & 4.4 & 41.9 & 56.9 & 29.9 & 6.1 & 60.5 \\
\hline \multirow{2}{*}{$\mathrm{Si}$} & $1.0 \mathrm{~g} / 1$ & 50.2 & 17.8 & 3.9 & 25.8 & 50.5 & 15.3 & 5.5 & 44.7 \\
\hline & $1.5 \mathrm{~g} / 1$ & 55.5 & 30.3 & 4.3 & 38.7 & 55.2 & 26 & 5.9 & 55.2 \\
\hline control & & 42.6 & ----- & 3.1 & ----- & 43.8 & --- & 3.8 & ------ \\
\hline LSD $p \leq 0.05$ & & 2.4 & & 0.059 & & 2.4 & & 0.06 & \\
\hline
\end{tabular}

Table ( 12 ) con.

\begin{tabular}{|c|c|c|c|c|c|c|c|c|c|}
\hline \multirow[b]{2}{*}{ substance } & \multirow[b]{2}{*}{ Con. } & \multicolumn{4}{|c|}{ Giza 129} & \multicolumn{4}{|c|}{ Giza 130} \\
\hline & & $\begin{array}{l}1000 \text { Grain } \\
\text { Weight }(g)\end{array}$ & $\begin{array}{c}\text { Increase } \\
\%\end{array}$ & $\begin{array}{c}\text { Plot } \\
\text { Weight (kg) }\end{array}$ & $\begin{array}{c}\text { Increase } \\
\%\end{array}$ & $\begin{array}{l}1000 \text { Grain } \\
\text { Weight (g) }\end{array}$ & $\begin{array}{c}\text { Increase } \\
\%\end{array}$ & $\begin{array}{c}\text { Plot } \\
\text { Weight (kg) }\end{array}$ & $\begin{array}{c}\text { Increase } \\
\%\end{array}$ \\
\hline \multirow{2}{*}{$\mathrm{KH}_{2} \mathrm{PO}_{4}$} & $50 \mu \mathrm{g} / \mathrm{ml}$ & 50.1 & 42.3 & 6.7 & 86 & 51.4 & 32.1 & 4.8 & 71.4 \\
\hline & $100 \mu \mathrm{g} / \mathrm{ml}$ & 55.2 & 56.7 & 7.6 & 111 & 57.35 & 47.4 & 5.7 & 103.6 \\
\hline \multirow{2}{*}{ CCNC } & $15 \mathrm{mg} / \mathrm{ml}$ & 48.6 & 38 & 6.2 & 72.2 & 49.6 & 27.5 & 4.2 & 50.0 \\
\hline & $30 \mathrm{mg} / \mathrm{ml}$ & 54.17 & 53.8 & 6.7 & 86 & 55.8 & 43.4 & 4.7 & 67.9 \\
\hline \multirow{2}{*}{ humic acid } & $1.5 \mathrm{~m} / 1$ & 46.9 & 33.2 & 6.1 & 69.4 & 45.9 & 17.9 & 3.8 & 35.7 \\
\hline & $2.5 \mathrm{~m} / 1$ & 50.9 & 44.6 & 6.4 & 77.7 & 53.2 & 36.7 & 4.5 & 60.7 \\
\hline \multirow{2}{*}{$\mathrm{Si}$} & $1.0 \mathrm{~g} / 1$ & 45.9 & 30.4 & 5.1 & 41.7 & 45.2 & 16.2 & 3.1 & 10.7 \\
\hline & $1.5 \mathrm{~g} / 1$ & 48.6 & 38 & 5.7 & 58.3 & 52.6 & 35.2 & 4.0 & 42.9 \\
\hline control & & 35.21 & -------- & 3.6 & ----- & 38.9 & ----- & 2.8 & ----- \\
\hline$\underline{\mathrm{LSD}} \mathrm{p} \leq 0.05$ & & 2.4 & & 0.06 & & 2.4 & & 0.059 & \\
\hline
\end{tabular}

Results in Table 13 at Sakha location with minor differences, where genotypes Giza 129 and Giza 130 were the most improving in 1000 grain weight than cvs.Giza
127 and Giza 128 . In contrast , improving plot weight was highly ranking in cvs.Giza 127 and Giza 128 than cvs.Giza 130 and Giza 129 compared to the control .

Table (13) : Effect of inducers on yield components on Giza 127and Giza 128, Giza 129 and Giza 130under field conditions during 2013/2014 growing season in Sakha .

\begin{tabular}{|c|c|c|c|c|c|c|c|c|c|}
\hline \multirow[b]{2}{*}{ substance } & \multirow[b]{2}{*}{ Con. } & \multicolumn{4}{|c|}{ Giza 127} & \multicolumn{4}{|c|}{ Giza 128} \\
\hline & & $\begin{array}{l}1000 \text { Grain } \\
\text { Weight (g) }\end{array}$ & $\begin{array}{c}\text { Increase } \\
\%\end{array}$ & $\begin{array}{c}\text { Plot } \\
\text { Weight (Kg) }\end{array}$ & $\begin{array}{c}\text { Increase } \\
\%\end{array}$ & $\begin{array}{l}1000 \text { Grain } \\
\text { Weight (g) }\end{array}$ & $\begin{array}{c}\text { Increase } \\
\%\end{array}$ & $\begin{array}{c}\text { Plot } \\
\text { Weight ( kg) }\end{array}$ & $\begin{array}{c}\text { Increase } \\
\%\end{array}$ \\
\hline \multirow{2}{*}{$\mathrm{KH}_{2} \mathrm{PO}_{4}$} & $50 \mu \mathrm{g} / \mathrm{ml}$ & 51.7 & 24 & 4.9 & 105 & 56 & 30.2 & 4.9 & 81.5 \\
\hline & $100 \mu \mathrm{g} / \mathrm{ml}$ & 65 & 56 & 5.8 & 152 & 59.3 & 37.9 & 5.5 & 103.7 \\
\hline \multirow{2}{*}{ CCNC } & $15 \mathrm{mg} / \mathrm{ml}$ & 50 & 19.9 & 3.9 & 52.6 & 55 & 27.9 & 4.1 & 51.8 \\
\hline & $30 \mathrm{mg} / \mathrm{ml}$ & 62 & 48.7 & 4.4 & 78.9 & 58.7 & 36.5 & 5.1 & 88.8 \\
\hline \multirow{2}{*}{ humic acid } & $1.5 \mathrm{~m} / 1$ & 49 & 17.5 & 3.7 & 42.1 & 46.7 & 8.6 & 3.9 & 44.4 \\
\hline & $2.5 \mathrm{~m} / 1$ & 60 & 43.9 & 4.1 & 63.2 & 56.7 & 31.9 & 4.9 & 81.5 \\
\hline \multirow{2}{*}{$\mathrm{Si}$} & $1.0 \mathrm{~g} / 1$ & 46.7 & 12 & 3.3 & 21.1 & 45.7 & 6.3 & 3.1 & 14.8 \\
\hline & $1.5 \mathrm{~g} / 1$ & 53.3 & 27.8 & 4 & 57.8 & 55.7 & 29.5 & 4.1 & 51.8 \\
\hline control & & 41.7 & ------- & 2.9 & ----- & 43 & ------ & 2.7 & ---- \\
\hline LSD $p \leq 0.05$ & & 1.7 & & 0.03 & & 1.7 & & 0.03 & \\
\hline
\end{tabular}

Table ( 13 ) : con.

\begin{tabular}{|c|c|c|c|c|c|c|c|c|c|}
\hline \multirow[b]{2}{*}{ substance } & \multirow[b]{2}{*}{ Con. } & \multicolumn{4}{|c|}{ Giza 129} & \multicolumn{4}{|c|}{ Giza 130} \\
\hline & & $\begin{array}{l}1000 \text { Grain } \\
\text { Weight (g) }\end{array}$ & $\begin{array}{c}\text { Increase } \\
\% \\
\end{array}$ & $\begin{array}{c}\text { Plot } \\
\text { Weight (Kg) } \\
\end{array}$ & $\begin{array}{c}\text { Increase } \\
\% \\
\end{array}$ & $\begin{array}{l}1000 \text { Grain } \\
\text { Weight (g) }\end{array}$ & $\begin{array}{c}\text { Increase } \\
\% \\
\end{array}$ & $\begin{array}{c}\text { Plot } \\
\text { Weight (kg) } \\
\end{array}$ & $\begin{array}{c}\text { Increase } \\
\% \\
\end{array}$ \\
\hline \multirow{2}{*}{$\mathrm{KH}_{2} \mathrm{PO}_{4}$} & $50 \mu \mathrm{g} / \mathrm{ml}$ & 55 & 38 & 6.9 & 81.6 & 63.6 & 47.9 & 7.3 & 69.8 \\
\hline & $100 \mu \mathrm{g} / \mathrm{ml}$ & 66.7 & 67 & 7.9 & 107.8 & 66.7 & 55.1 & 7.9 & 83.7 \\
\hline \multirow{2}{*}{$\mathrm{CCNC}$} & $15 \mathrm{mg} / \mathrm{ml}$ & 51.7 & 29 & 6.3 & 65.8 & 63.3 & 47.2 & 6.9 & 60.5 \\
\hline & $30 \mathrm{mg} / \mathrm{ml}$ & 65 & 63 & 6.8 & 78.9 & 65.6 & 52.6 & 7.1 & 65.1 \\
\hline \multirow{2}{*}{ Humic acid } & $1.5 \mathrm{~m} / 1$ & 50 & 25 & 6 & 57.9 & 53.3 & 23.9 & 6.1 & 41.9 \\
\hline & $2.5 \mathrm{~m} / 1$ & 56.7 & 42 & 6.6 & 73.7 & 60 & 39.5 & 6.7 & 55.8 \\
\hline \multirow{2}{*}{$\mathrm{Si}$} & $1.0 \mathrm{~g} / 1$ & 43.3 & 8 & 4.9 & 28.9 & 51.3 & 19.3 & 5.9 & 37.2 \\
\hline & $1.5 \mathrm{~g} / 1$ & 55 & 15 & 5.6 & 47.4 & 58.3 & 35.6 & 6.3 & 46.5 \\
\hline control & & 40 & ------- & 3.8 & ------ & 43 & ------ & 4.3 & ------ \\
\hline LSD $p \leq 0.05$ & & 1.7 & & 0.03 & & 1.7 & & 0.03 & \\
\hline
\end{tabular}




\section{DISCUSSION}

Application of Mono potassium phosphate $\mathrm{KH}_{2} \mathrm{PO}_{4} \quad(50 \quad \& 100 \mu \mathrm{g} / \mathrm{ml}) \quad$, Chitosan clay nanocomposite (ccnc) $(15 \& 30 \mathrm{mg} / \mathrm{ml})$, Humic acid(1.5 $\& 2.5 \mathrm{~m} / \mathrm{l})$ and Sodium meta silicate(Si) ( $1 \& 1.5 \mathrm{~g} / \mathrm{l})$ reduced the injurious effect of net blotch ( $\mathrm{BNB}$ ) on barley where Mono potassium phosphate and Chitosan clay Nano composite were the most effective while Humic acid was moderately effective .

Data of the present study showed that, under greenhouse and field conditions all treatments and their concentrations were significantly reduced disease severity and consequently improving the chemical composition of grains and yield component in barley varieties as compared to the untreated control. In this research Chitosan clay nano composite and $\mathrm{KH}_{2} \mathrm{PO}_{4}$ treatment showed the higher efficacy in reducing net blotch disease may be due to increasing the activites of peroxidase and $\beta-1,3$ gluconase moreover it is associated with increase in activites of many class of PR proteins (Abou-Taleb .2001 and Mosa ,2002) .

Glucanass are known to play a direct role in plant defence mechanism by hydrolyzin structural components from fungal cell wall and act as elicitors which lead the accumulation of a phytoalexin and glyceollin (Sharp et al.,( 1984) .Also , peroxidase play important role in oxidation process of phenol compounds to oxidized products (quinones ) which may limit the fungal growth . peroxidase produce free radicals and hydrogen peroxide which are toxic to many microorganisms Pena and Kue (1992).

The 1000 Kernal weight is a highly important parameter for yield and technological quality in barley.Przulj and Momcilovic(2006)Data in this study showed that all treatment improving crop parameters , In this respect $\mathrm{KH}_{2} \mathrm{PO}_{4}$ following chitosan were the most effective treatment to increase 1000 kernel weight and plot weight where efficacy of $\mathrm{KH}_{2} \mathrm{PO}_{4}$ against plant infection to trigger plant defense through a process involving the consequent of calcium from host issue , elicits the release of signal triggers of plant response and makes the plant more resistance and more responsive after subsequent infection (Gottstein and Kuc, 1989 , Mucharromah and Kuc, 1991 ). Moreover $\mathrm{KH}_{2} \mathrm{PO}_{4}$ led to increase synthesis of host metabolites such as phytoalexins and their production could be induced by many chemicals and increase of enzymes activities and total phenols Nighat et al.,(2011) .

chitosan also used in plant disease control as a powerful elicitor . Its direct toxicity remains dependent on properties .chitosan is known to act as potent inducers, enhancing a battery of plant responses both locally around the infection sites and systemically to alert healthy parts of the plant . These include early signaling events as well as the accumulation of defense - related metabolites and proteins such as phytoalexins and PR- proteins (El Hadramiet al., 2009 and Hammerschmidt,1999) . It can be formation of physical barrier preventing the pathogen from spreading and invading other healthy tissues (El Hadramiet al., 2009) . Chitosan is applied to plants to prevent diseases because it can chelate nutrients and minerals ( i.e., fe . cu ) preventing pathogens from accessing them (Bornet ,2007) . It is activity of peroxidase and $\beta 1-3$ gluconse which realized in this study by increasing the activity of both enzymes due to chitosan treatment, it is agreement with El Hadrami et al., (2010) whow found that , chitosan elicited peroxidase and polyphenoloxyidase activities and increased the level of phenolic compounds.

Data obtained showed that there were negative correlation between disease severity and 1000 kernel weight, plot weight and total fiber where varietieshulless barley Giza 129 and Giza 130 were moderate resistance to net blotch than hulled barley Giza 127 and Giza 128 thus the highest value of these trails were in hulless barley than hulled barley, so the yield components depend on disease severity and varieties, this finding is in harmony with Gaunt (1980) who described that, the yield components affected by Pyrenphorateres depend on severity and duration of disease on barley in addition to complex physiologic process of the host. Similar results have been demonstrated early byJordan (1981) who reported that, satisfactory yield can still be obtained with genotypes possessing in complete resistance .

A previous study showed that six- rowed genotype may possess higher level of resistance than two- rowed .This similarlywith Tekauz, (2002) who reported that six-rowed genotypes are more resistant than two- rowed varieties.

The opinion of many authors that the chemical composition of barley grain is modified not only by weather conditions but also it depend on the plant genotypes Noworolink (2004) .

Chitosan following by humic acids ( HA) were the most effective for improving chemical composition as total fiber. where efficacy of humic acid (HA) in overcoming the harmful effects of barley net blotch ( BNB) plant may be due to the increase in chitinase activity and stimulation plant growth through increased cell division, as well as optimized uptake of nutrients and water ( Atiyeh et al., 2002 and Chen et al., 2004 ) also, regulate hormone level, improve plant growth and enhance stress tolerance (Piccolo et al., 1992). HA is a suspension, based on potassium humates, which can be applied successfully in many areas of plant production as a plant growth stimulant or soil conditioner for enhancing natural resistance against plant diseases and pests (Scheuerell and Mahaffee, 2006) which consequently increase yield of plant . Foliar application of (HA) consistently enhanced antioxidants such as $\alpha$-tocopherol , $\beta$-carotene , superoxide dismutases, and ascorbic acid concentrations in turf grass species (Zhang, 1997). These antioxidants may play a role in the regulation of plant development, flowering and chilling of disease resistance (Ziadi et al., 2001). 
finally ,In short, use of chemical inducers ,as safe substance, may improve yield component and reduce disease severity of net blotch in barley plants .

\section{REFERENCES}

Abou- Taleb, M.A.(2001). Biochemical changes associated with the application of some resistance - inducing compounds for controlling powdery mildew of cucumber. Egypt .J. Appl.Sci., 16 :387405 .

A .O . A .C (2005) . Official methods analysis of Association of Official Agriculture Chemists . 18 th End Washing D. C.

Atiyeh , R.M.; Lee, S.; Edwards, C.A.; Arancon, N.Q. and Metzger, J.D. (2002). The Influence of humic acids derived from earthworm proceed organic wastes on Plant growth . BioresourceTechnology , 84: 7-14.

Biles , C.L. and Martyn , R.D. (1993) . Peroxidase ,polyphenoloxidase and shikimate Dehydro genisozymes in relation to tissue type, maturity and pathogen induction of Watermelon seeding . Plant physiol .Biocherm., $31:$ 499-506.

Bornet, A. T .( 2007). Chitosan , chitin - glucan and chitin effect on minerals ( iron, lead, cadmium) and organic ( ochratoxin A) contaminants in wines . Eur. Food Res. Technol.226(4), 681-689.

Boungab,K; Belabid, L. ; Fortas, Z.; Bayaa ,B. (2012).Pathotype diversity among Algerian isolates of Pyrenophora teres $f$. teres .Phytopathol .Medit ., 5 (3), 577-586

Chen, Y., Nobili ,M. De and Aviad, T. (2004). Stimulatory effect of humic substances on plant growth. In: Magdoff F, Weil RR, editors. Soil organic matter in sustainable agriculture . Boca Raton:CRC Press : 103-130

El Hadrami , A.; Lorne R. A ; El Hadrami , I. and Fouad ,D. (2009) . Suppression of induced plant defense responses by fungal pathogens. In Molecular Plant Microbe Interactions ;Brisson , N., Daayf, F., Eds; CABI: Wallingford , UK, Chapter 10 , pp. 231-268.

El Hadrami , A.; Lorne R. A ; El Hadrami , I. and Fouad, D. (2010).Chitosan in plant protection .Marine Drugs ,8 : 968-987 ..

Eyal , Z; Scharen ,A.L.; Prescott ,J . M. and Van Cinkel, M .( 1987). The septoria disease of wheat; concepts and methods of diease management . Mexico D.F.,CIMMYT, PP.46 .

Faten El-Nashar, K. (1983) . Net blotch disease of barley caused by Drechslera teres( Sacc.) Shoem . M.Sc. Thesis, Fac. Of Agric. Cairo Univ.

Gary , E . V. and Robert , M .G.(2004) Systemic Acquired Resistance and Induced Systemic Resistance in Conventional Agriculture. Crop Sci.,44: 1920- 1934
Gaunt, R . E.(1980). Phsiological basis of yield loss. Pages 98-111 in : proceeding of E.C Stakman Commemorative Symposium on Assessment of Losses which constrain production and crop improvement in Agriculture and Forestry . Univ. Minn. Agric. Exp . Stn. Misc. Publ.7.

Gottstein , H.R. and Kuc, J. (1989 ). Induction of systemic resistance to anthracnose in cucumber Byphosphales. Phytopathology, 79: $176-179$.

Gupta, S .and loughman, G.J .( 2003 ) . RCM Lance . Aust. J. Agr. Res., 54,1379-1386.

Hammerschmidt, R.(1999) .Phytoalexins : what have we learned after 60 years? Ann Rev. Phytopathol. 37, 285-306.

Jordan , V. W. L (1981) .A etiology of barley net blotch caused by pyrenophora teres and some effects on yield . Plant Pathology 30: 77-87.

KunzhengCai, D. G. and Shiming,L ( 2009) . Probing the mechanisms of silicon - mediated pathogen resistance .Plant Signal Behav.4 (1) :1-3 .

Large, E.C.(1954). Growth stages in cereals .IIIustration of the Feek'sScale . Plant Pathol.,3:123-129.

Mosa , A.A. (2002). Induced resistance in rice against blast disease using abiotic and biotic agents. Ann. Agric. Sci.Ain Shams Univ.,47:993-1008.

Malcolmson, L., Nowkirk , R. and Carson, G. (2005) . Expanding opporatunities for barley food and feed through product innovation .feed and quality $; 18^{\text {th }}$ National American Barley Research Workshop $4^{\text {th }}$ Canadian Barley symposium . pp : 2-4 .

Mucharromah , E. and Kuc, J. (1991) . Oxalate and phosphates induced systemic resistance against Disease caused by fungi, bacteria and viruses in cucumber. Crop Protection, 10:265-270.

Nighat, S ; Zahid , M.H; Ashfaq, S. and Jamil , F.F.( 2011). Induced systemic resistance in Chickpea against Ascochyta blight by safe chemicals . Pak. J.Bot., 43(2):1381-1387.

Noworolnik, K. (2004).nitrogen fertilization efficiency of buckwheat grown at various soil conditions . the $6^{\text {th }}$ international symposium on buckwheat.Current Advances in Buckwheat Research , 601-604.

Pan, S.Q., Ye, X.S., and Kuc, J. (1991) .Association of $\beta-1,3$ glucanase activity and isoform Pattern with systemic resistance to blue mold in tobacco induced by stem injection with Peronosporatabacinaor leaf inoculation with tobacco mosaic virus. Physiol . Mol. PlantPathol., 39:25-39.

Pena , M. and Kuc, J . A.(1992) . Peroxidase generated hydrogen peroxidase as a sources of antifungal activity in vitro and on tobacoo leaf disk .Phytopathology , 82: 696-699 .

Piccolo, A.; Nardi, S. and Concheri , G. (1992) .Structural characteristics of humic substances As regulated to nitrate uptake and growth regulation in plant system. Soil Biochem ., 24: 373-380 . 
Poehlman , J. M .(1985) . Adaptation and distribution. Pp.1-17. In: Barley .Rasmusson , D. C.(ed.). Agronomy Monograph No. 26, ASA . Madison. WI.

Przulj,N.andMomcilovic,V.(2006).Barly breeding for yieldand quality.GlasnikZastiteBilja., 29: 1, 49-57.

Reuveni, M. andReuveni, R., (2001). Monopotasssium Phosphate fertilizer (peak)-a component in integrated Control of powdery mildews in fruit trees and grapevines . Acta. Hort., 594: 619- 625.

Scheuerell, S.J. and Mahaffee, W.H. (2006).Variability associated with suppression of graymould (Botrytis cinerea) on geranium by foliar applications of no aerated and aerated compost Tekas. Plant Dis. , 90: $1201-1208$

Sharp , J. K., Valent , B. and Albersheim, P. (1984). Purification and partial characterization of a $\beta-$ glucan fragment that elicits phytoalexin accumulation in soybean . J. Biol .chem. 259, 11312 - 11320.

Steffenson, B. J., Webster, R. K., and Jackson , L.F. ( 1991). Reduction in yield loss using incomplete resistance to Pyrenophorateresf.teresin barley plant Dis. 75:96-100

Tekauz A (1978): Incidence and severity of net blotch of barley and distribution of Pyrenophora teres biotypes in the Canadian prairies in 1976.Canadian Plant Disease Survey 58, 9-11.

Tekauz, A (2002).Spot blotch( Cochlibolussativus) infection response in selected North American barley cultivars at the seedling and adult- plant stages . Pp. 428-435.In: Proceedings of the Second International Workshop on Barley Leaf Blight . ICARDA, Aleppo, Syria .
Tekauz, A (2003). Spot blotch caused by Bipolaris sorskiniana : an emerging problem in barley In western Canada. In : proceedings of 8 th International Congress of plant Pathology Christchurch, New Zealand .Pp. 103.

Towsend , G. K. and Heuberger, T.W. (1943). Methods for estimating losses caused by disease experiments . Plant Dis. Rept., 27: 340-343.

Yildirim,E., (2007 ). Foliar and soil fertilization of humic acid affect productivity and quality of Tomato.Acta Agric. Scandinavia . Section B. Soil and Plant Sci., 57 ( 2): 182-186.

Yitbarek , S.and Wudneh ,E. . (1985). Preliminary studies on the yield losses due to net blotch in Barley ,pp 47-52 In Proceedings of the $10^{\text {th }}$ EPC annual meetings . Addis Ababa. Ethiopia .

Yamaguchi, I. ( 1998 ) . Activators of systemic acquired resistance . In: Fungicidal activity: Chemical and biological approaches to plant protection .Hutson D.H. Miyamoto J., eds. New York :Wileyev\& s611s Inc., pp. 193-219.

Zhang, X. (1997) . Influence of plant growth regulator on turfgrass growth antioxidant status, And drought tolerance . Ph.D. Thesis, Fac. Of Virginia polytechnic, 131p.

Ziadi , S.; Barbedette , S., Godard, J.F.; Monoti, C.; Corre , L.E.D.; Silue, D. and Lecorre, D. (2001) . Production of pathogenesis related protein in the cauliflower (BrassicaoleaceaVar. botrytis) downy mildew (Peronosporaparasitica) pathosystem treated with Acidbenzolar -5- methyl. Plant Pathol.50 (5):579-586.

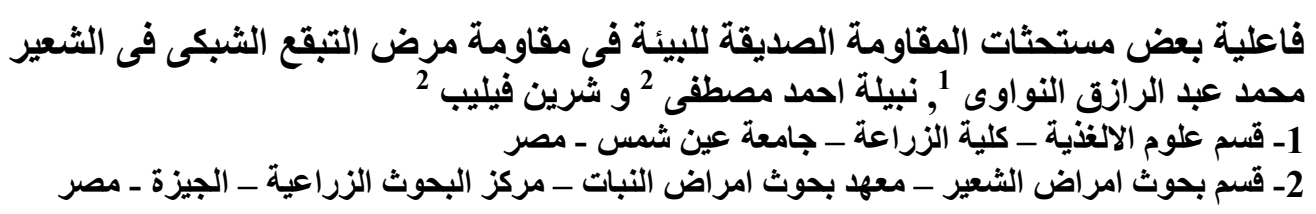

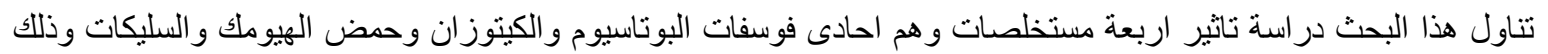

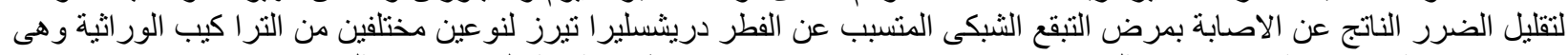

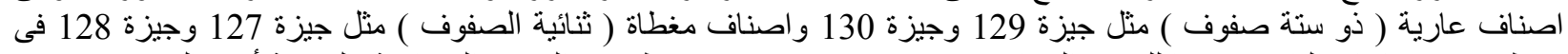

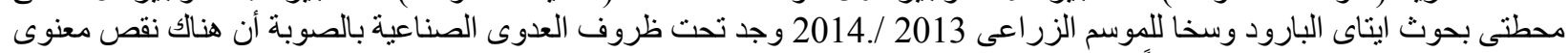

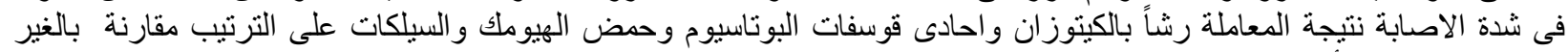

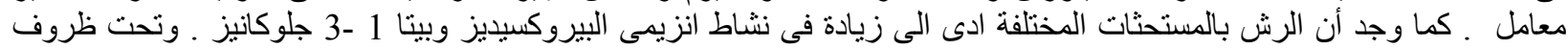

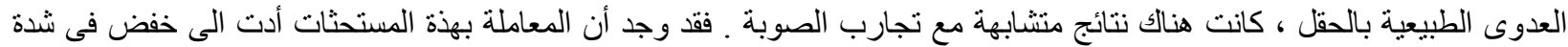

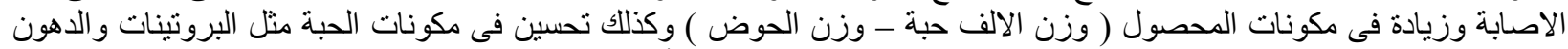

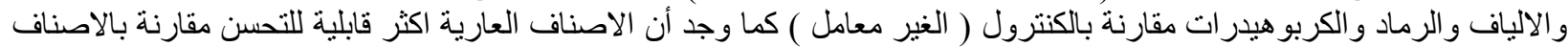

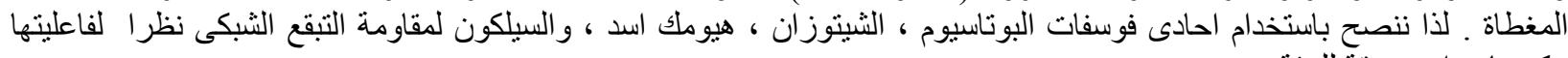
وكونها مو اد صديقة للبيئة . لذاة بانية 
J.Agric.Chem.and Biotechn., Mansoura Univ.Vol. 7 (6): 153 - 161, 2016 